\title{
A Safe, Efficient and Integrated Indoor Robotic Fleet for Logistic Applications in Healthcare and Commercial Spaces: The ENDORSE concept.
}

\author{
Nacim Ramdani*, Andreas Panayides ${ }^{\dagger}$, Michalis Karamousadakis ${ }^{\ddagger}$, Martin Mellado ${ }^{\S}$, \\ Rafael Lopez ", Christophoros Christophoroull, Mohamed Rebiai**, Myriam Blouin**, \\ Eleftheria Vellidou ${ }^{\dagger \dagger}$ and Dimistris Koutsouris ${ }^{\dagger \dagger}$ \\ *Univ. Orléans, INSA CVL, PRISME EA 4229, F45072 Orléans, France \\ Email: nacim.ramdani@univ-orleans.fr. \\ ${ }^{\dagger}$ Department of Computer Science, University of Cyprus, 1678 Nicosia, Cyprus \\ $\ddagger$ SingularLogic, Achaias 3 \& Troizinias, 14564 Nea Kifisia, Greece \\ §Instituto ai2, Universitat Politècnica de València, 46022 Valencia, Spain \\ IR\&D Department, Robotnik Automation, 46988 Paterna, Spain \\ $\|_{\text {Citard Services, } 2064 \text { Strovolos, Cyprus }}$ \\ ${ }^{* *}$ Stream Vision, 75009 Paris, France \\ †† Institute of Communication and Computer Systems, 10682 Athina, Greece
}

\begin{abstract}
Hospitals are rightfully considered a field of indoor logistic robotics of high commercial potential. However, today, only a handful of mobile robotic solutions for hospital logistics exist that have failed to trigger widespread acceptance by the market. This is because existing systems require costly infrastructure installation, they do not easily integrate to corporate IT solutions, are not adequately shielded from cybersecurity threats, and as a result, they do not fully automate procedures and traceability of the items they carry. Moreover, existing systems are limited on scope, focusing only on delivery services, and hence do not provide any other type of support to the medical and nursing staff. ENDORSE system will address the aforementioned technical challenges and functional limitations by pursuing four innovation pillars: (i) infrastructure-less multirobot indoor navigation; (ii) advanced Human-Robot Interaction (HRI) for resolving deadlocks and achieving efficient sharing of space resources in crowded environments; (iii) deployment of the ENDORSE software as a cloud-based service facilitating its integration with corporate software solutions, complying with GDPR data security requirements; (iv) reconfigurable and modular hardware architectures so that diverse modules can be easily swapped. ENDORSE functionality will be demonstrated via the integration of an e-diagnostic support module for vital signs monitoring on a fleet of mobile robots, facilitating connectivity to cloud-based Electronic Health Records (EHR), and validated in an operational hospital environment for realistic assessment.

Index Terms - Intelligent robotics, indoor navigation, e-Health, medical informatics, embedded systems, hardware platforms.
\end{abstract}

\section{INTRODUCTION}

Autonomous mobile robots for transport of goods and materials are nowadays a well-established solution in large

This project has received funding from the European Union's Horizon 2020 research and innovation programme under the Marie Skłodowska-Curie grant agreement No 823887.

RISE MSCA ENDORSE project www.endorse-project.eu. industrial infrastructures and transit warehouses, where they offer significant economic and administrative advantages. In contrast, in spaces such as hospitals, nursing centers, hotels, museums, malls, commercial spaces, offices and retail stores, which herein for economy of space we call commercial spaces, the use of autonomous robots is very limited. The use of existing robotic systems designed for large industrial and warehouse spaces is not applicable to indoor commercial spaces because of the different specifications and different constraints. A fundamental difference is that large industrial and warehouse spaces are characterized by very structured and predictable environments where robots move in predefined paths and interaction with humans can easily be avoided. Contrary to the industrial warehouses, commercial spaces are less structured, the environment is much more dynamic and the interaction with humans is frequent. Also, the load specifications are different and the interior architectural specifications are much more constrained.

Automation of the supply chain in commercial spaces could have a high economic impact, given the large number of daily transfers taking place in these premises. For example, in a hospital or in a health care center, several tenths of person months are spent annually on transfer of goods, linens, biological samples, medical equipment, pharmaceuticals, mail parcels, and medical waste, amounting to more than 850 man hours per week in a 500 beds hospital [1]. These transfers are currently carried out manually by pushing carts for long distances by nurses or carers. This is a no-added-value job which deprives the patients from valuable nursing duties. The same arguments apply to the majority of commercial spaces listed earlier. In addition, automation of the supply chain will enable enhanced traceability of the goods and of the products 
from the time of the request until the moment of delivery to the recipient. In other words, a well programmed logistics system will improve the services offered to the end-user (i.e., the hotel customer or the patient and the carer in a hospital), by reducing the response times for each request. Hence, the indoor supply chain is an application that calls for automation and at the same time has high commercialization potential because the involved corporations and organizations hold a huge part of the market, they have specific practical problems, and seek specific solutions for which they are willing to pay.

\section{BACKGROUND AND OBJECTIVES}

Indoor spaces in hospitals and healthcare centers offer advantages that would facilitate the deployment of mobile robots. They are required by law to meet stringent building codes and therefore, from a robotics perspective, the navigation space exhibits some structure which moderates the technical challenges, e.g., with respect to home environments. Moreover, most commercial spaces have reliable and predictable communications infrastructure, e.g., Wi-Fi and cellular connectivity, since it is required for smooth business operation [2]. Thus, spaces in hospitals and healthcare centers are rightfully considered the next great field of robotic deployment, following the industrial spaces. Despite these advantages, today, only a handful of solutions exist for hospital and healthcare spaces -unlike the case of industrial spaces- and these solutions do not trigger widespread acceptance by the market. This is because they require costly and time-consuming localization infrastructure, e.g., significant peripheral sensor installation, mapping, etc.; they do not easily integrate to corporate IT solutions; as a result, they do not address satisfactorily data protection and cybersecurity threats; they are not adaptable and reconfigurable and therefore are limited to a single type of service. Existing solutions for small and medium commercial spaces such as hospitals and healthcare centers, developed by Aethon (aethon.com) and SwissLog (www.swisslog.com) are very expensive, they cost more than 75,000 euros plus installation costs, and exhibit operational limitations. They lack motion flexibility and therefore the design and implementation of their route becomes challenging in small spaces; they are not customizable and modular and therefore perform no tasks other than transfer of goods; hence they lack the capability to replace their carts with other functional units such as an ehealth diagnostic station or a UV-light sanitizer. These systems are difficult to integrate with existing Enterprise Resource Planning (ERPs) and business intelligence systems typically installed in healthcare buildings, and therefore the data they collect and the tasks they execute are not registered in the corporate records and data management system. Cybersecurity threat is another deterrent factor, especially in cases where humans are involved.

The solution to the aforementioned limitations, and the overall goal of the ENDORSE concept, is to design and develop integrated logistic robotic systems that will enable efficient, cost-effective and safe indoor logistic services for hospital spaces.
The specific objectives of ENDORSE are described next:

1) To develop a cost-effective logistics robotics system for healthcare indoor environments. A key point for the achievement of this goal is the minimization of installation time and of the cost of the system.

2) To push forward the frontier of Human-Robot Interaction (HRI), especially for the cases where robot should safely navigate in crowded spaces, resolving deadlocks that involve humans.

3) To develop robust localization methods for infrastructure-less indoor robotic fleet localization and supervision, which will result in installation time minimization.

4) To introduce novel cloud services that bring together solutions from healthcare, robotics, and logistics, offering new business models for indoor logistics and high data security standards.

5) To develop an integrated mobile e-diagnostic station, which will endow modularity to the ENDORSE system, and will facilitate a mobile e-diagnostic service.

The objectives will be achieved by tackling specific scientific and technological challenges through cross-sector collaboration taking advantage of complementary competences on the fields of robotics, machine vision, logistics, IT and product design. The proposed solution will be modular and reconfigurable and thus applicable to different fields and applications of commercial spaces, while it will focus-in on the needs of the healthcare sector. The developed solution will be piloted in healthcare center Fundació Ave María in Sitges, Spain.

\section{CONCEPT DESCRIPTION}

The ENDORSE project will realize the aforementioned objectives by relying on four main innovation pillars:

\section{A. Infrastructure-less multi-robot navigation}

Novel internet-based indoor localization technologies will be developed for this purpose. These will enable multi-robot navigation requiring minimum or no installation of infrastructure on building walls, ceilings and floors, hence ensuring minimum sensors and communications buses. A typical sensor installation costs over 10,000 euros and requires several days for installation and integration. Hence, this approach will considerably reduce the cost and the time needed for infrastructure installation of indoor logistic systems by fusing state-of-the-art wireless sensors on board the robot with robust Simultaneous localization and Mapping (SLAM) algorithms. A fundamental problem is that there is currently no open-source platform to seamlessly integrate fingerprint measurements from different types of indoor (and robot on-board) localization technologies (e.g., Wi-Fi, BLE, UWB, etc.) [3]. Our approach will be to extend the open-source Anyplace (anyplace.cs.ucy.ac.cy) architecture with components and specialized localization algorithms, which will enable fine-grain infrastructure-free localization using Internet of Things (IoT) -enabled devices. We will particularly redesign the algorithmic components 
necessary for indoor localization, indoor localization accuracy estimation [4], API channel optimization techniques [3], and visual analytic cues necessary to provide a rigorous Fingerprint Management Studio for crowd-sourced indoor signals. The infrastructure-less multi-robot navigation will be implemented by a software architecture that will combine ROS packages for navigation and path planning implemented onto the commercially available Robotnik (www.robotnik.eu) RB1-base as shown in Fig. 1, and Java and Javascript modules for the GUI and the fleet management system implementation.

\section{B. Human-aware navigation for indoor logistic applications}

The robotic revolution is as much about getting the machines to adapt to humans as it is about humans adapting to the machines. This convergence requires intuitive HRI so that untrained personnel in a Healthcare center can smoothly interact with the robot to avoid deadlocks, while the robot itself is also capable of adapting to the crowd and conducting human-aware navigation. Security aspects also play an important role, so that logistic tasks are addressed to the authorized personnel, leveraging communication and message activation systems that further minimize stress and maximize the comfort of people interacting with the robot. These functionalities will be developed and accommodated by the ROS perception packages that run on-board the robot.

\section{Reconfigurable and modular hardware architectures}

One of the major innovations and tangible technical outcomes of the project will be the development and testing of the modular interfaces and of the e-diagnostic mobile station integrated on-board the mobile robot and tested in a healthcare environment. This will require research and development in both non-invasive sensors/devices (ECG, blood pressure, oximeter, temperature) and dedicated software applications. To this end, modular hardware interfaces (mechanical, electrical and data interfaces) mounted on the chassis of the Robotnik RB1-base robot will be developed (see Fig. 1), so that different hardware-based functional modules can be easily swapped. This would be the first time that a medical diagnostic functionality is integrated on a mobile robot. The integrated robot will serve as an e-diagnostic mobile station, which will be able to produce a diagnostic exam report that will be then securely forwarded to update and complement the patients' cloud-based Electronic Health Records (EHR). The software of the e-diagnostic mobile station will run on-board the robot.

\section{Cloud based services}

Cloud computing delivers ubiquitous, $24 / 7$ access to software-as-a-service (SaaS) solutions, capable of adapting to varying degrees of user load. Moreover, cloud services must guarantee what is known as a Service Level Agreement (SLA). Typically, SLAs have been customarily specified as some percentage time during which the functionality of the service is available. Guaranteeing the SLA of a service is, thus, intrinsically related to the management of the service

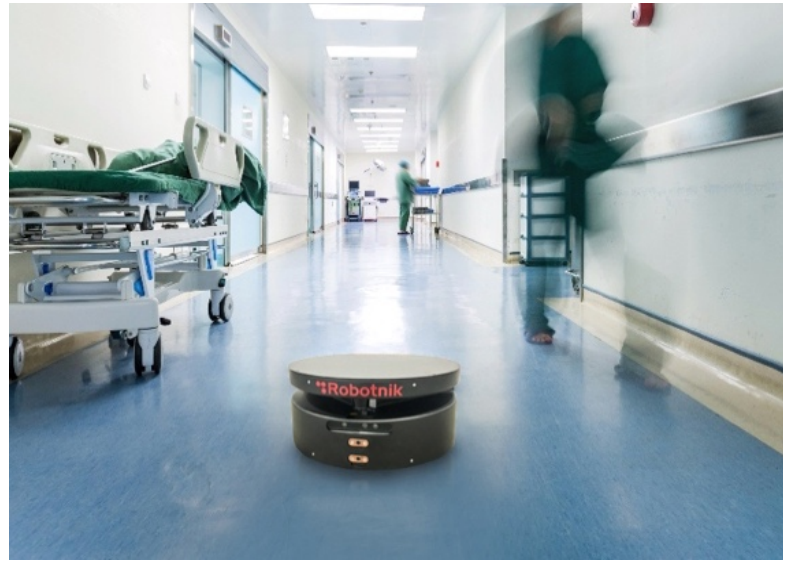

Fig. 1. The commercially available Robotnik RB1-base robot navigating in a healthcare environment

life cycle, and the events driving it: initial deployment, reconfigurations, upgrade deployment, fault handling, re-scaling. Automation makes it possible to manage the data centres so that they deliver portions of themselves (in the form of virtual machines and networks) in a cost-effective manner. Cloudbased applications will offer to the enterprise users a high memory service, data sharing and security. Cloud servers will be able to connect with different individual applications and enterprise legacy systems. The cloud service proposed will contain a repository of algorithms, which will be fed by the data gathered through the cloud. The algorithms in the cloud will allow supporting the management of fleet, including the assignment of tasks to the robots, the queuing functionalities, the generation of dynamic routing and traffic control of the robots, and the supervision the fleets task execution. Developing a cloud oriented software platform will facilitate the development of APIs for integrating ENDORSE software with corporate ERP solutions and heath record systems that are often provided as a service in a Cloud environment. Most importantly the ENDORSE applications will comply with the high safety standards for data sharing and data storage which are offered by the Cloud infrastructure. Cloud-based services will run on a cloud server and will be interfaced by the user through a web-based application.

\section{Functional Architecture}

The aforementioned concept is implemented by the system functional architecture depicted on Fig. 2.

During system operation, five levels of supervision and control naturally emerge, dictated both by time requirement, i.e. the loop-rates, and the level of abstraction of the signals which are involved. This leads to hierarchical architecture composed of levels for controlling motors of the wheels, the robot modules, behaviors and tasks, and is described next in a bottom-up sequence.

At the lowest level of hierarchy is the Signals level which involves the process of raw signals and motor control such as the wheel motors. 


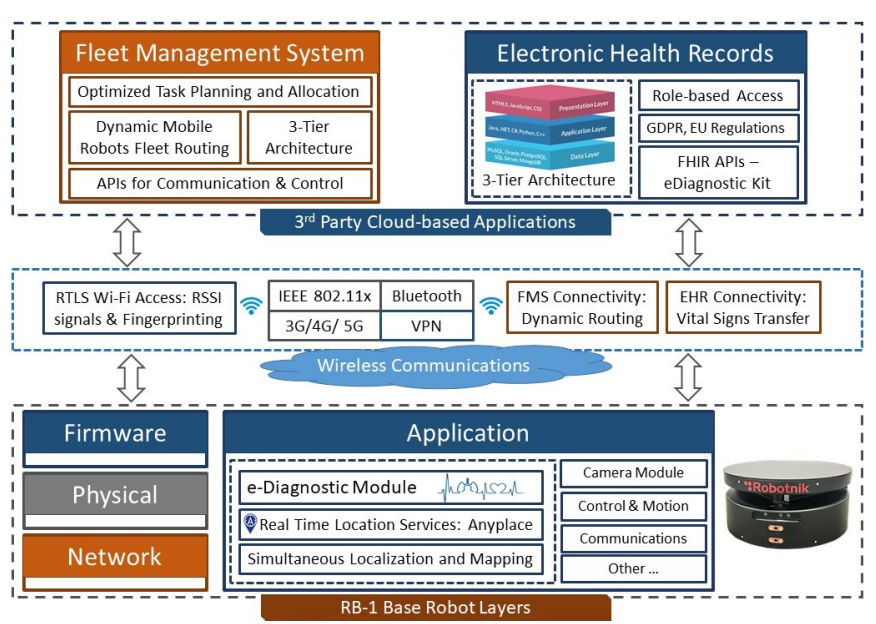

Fig. 2. An abstract system architecture of the ENDORSE solution.

The second level of hierarchy is that of the Motion level. This represents information in terms of the module and its environment. Each module's controller accepts asynchronous commands to move and turn with respect to a common reference frame.

The next level is the Planning level controller. This involves robot's actions and reactions; it is responsible for on-line path and motion planning, and transforms from the current state of the robot to a new state.

The next level is the Task level where a software platform of the Fleet Management System runs, i.e., task planner, robot dynamic routing, multi-map manager, elevator controller, etc.

Finally, the highest level of the hierarchy is the GUI level. The GUI level and the Task level can be grouped since, these will run on the Cloud, whereas all the rest will run on board.

\section{Technological Solutions}

\section{A. Cloud-based services}

In the project, a creation of an API framework will be accomplished, which will integrate logistic robotics into the corporate IT environment, and enable end-to-end automation (automatic traceability of the transferred goods, update of the ERP databases when transactions are carried out, etc.). Moreover, communication and security standards will be investigated in order to securely transfer data from the robot to the patients cloud-based EHR. To realize this task, ROS-based software integration will be pursued, where all system modules will be represented by ROS packages and ROS nodes. NonROS modules (i.e. Java or JavaScript applications) will have APIs that create objects that can be translated by ROS bridge package into ROS standard objects (topics, services, actions, etc.).

Due to its integrative nature this task will be implemented iteratively and will be tightly inter-leaved with safety humanrobot interaction protocols. An initial integrated software prototype will be developed given the prioritized system requirements and the output of the implementation. Then this initial prototype will be integrated with the Cloud-based services. The evaluation will be done in an iterative manner resulting in intermediate versions. The special requirements of the healthcare development are already identified and the development process will use them as a solid basis.

A diagnostic support module and the Electronic Health Records (EHR) interfacing will be also included. This will require research and development in both non-invasive sensors/devices (ECG, blood pressure, oximeter, temperature) and dedicated software applications. These sensors could be based on embedded microcontrollers processing units, with 32-bit ARM-Cortex SoC. This chip also will incorporate a complete communication layer that make the device compliant with BLE4.2, BLE5.0, IPv6, ANT/ANT+ and NFC-A tag protocols. In accordance of other magnitudes, the device could be equipped with a 9-Axis Inertial Measurement Unit (IMU) that makes possible monitoring the activity and real-time orientation. When the diagnostic support module is mounted on the robot chassis the overall robot will serve as an ediagnostic mobile station, which will be able to produce a diagnostic exam report that will be securely forwarded to update and complement the patients cloud-based EHR.

\section{B. Internet-based indoors localization technologies}

The ENDORSE project's localization solution will be geared towards infrastructure-less multi-robot navigation. In that context, the robustness of SLAM techniques used by ENDORSE will be enhanced, so that the navigation capabilities of the fleet will be fostered.

Standard robot localization or location tracking approaches often rely on state estimation algorithms working in a stochastic framework; they usually show weak performance when in presence of data outliers, systematic errors or invalid model assumption. Besides, most of these approaches require initial robot position to be known at least approximately, hence cannot solve the lost robot problem. We will develop a more robust and effective alternative, which is offered by set membership estimation and data fusion techniques that work in the bounded-error framework [5], [6]. These techniques can operate efficiently with sparse, asynchronous and heterogeneous measurements while being robust to the presence of non-consistent measurements, inaccuracy in environment modelling, and drift and inaccuracy in the robot evolution model. Furthermore, they can naturally track multihypotheses about robot pose, hence offer a solution technique for the robot global localization problem [7], [8]. Fostering on these approaches, effective data fusion and state estimation algorithms will be develloped for indoor robust localization and mapping-update in actual environments.

Besides, a comparison and a selection of on-board sensors, that are employed for localization, will take place, in order to improve accuracy and stability of global localization, since localization techniques fail and become less accurate in large spaces where Lidar or RGB-D have travel long distances, and reduce the computational burden of SLAM, which might result in deployment of less expensive hardware. To this end, the key tradeoffs will be identified on the requirements both on the 
type and nature of the sensors embedded or installed on-board the robot and on the environment. IMU will be considered for dead reckoning, cameras for line or feature following, as well as wheels encoders. Moreover, the use of RSSI measurements will be investigated (existing Wi-Fi beacons or deploy BLE or UWB). Ideally, only on-board sensors will be utilized hence minimizing installation time of the ENDORSE system. However, alternative solutions, using barcodes or QR codes on the wall in well-chosen areas, will also be deployed, to make localization easier for the case of docking.

\section{Electronic Health Records}

The European Union identified the development of eHealth [9] and mHealth [10] systems and services as a top priority for the advancement of the quality of healthcare provision, leading to the advancement of patients' quality of life, while saving significant healthcare expenditures. Placing the citizen at the center of innovations, the development of cloud-based EHR systems, facilitating the complete patient's medical history, will allow medical experts for more informed, timely decisions, hence advancing the level of current clinical practice. For achieving such a radical transformation, it is essential for different healthcare systems to be able to communicate and share information. In other words, install the necessary interoperability mechanisms that will allow medical experts from different specialties, practicing medicine in different hospitals, and using different systems, to connect to a patient's EHR and update it with the relevant clinical information. Towards this direction, the European Commission Decision 2015/1302 of July 2015 recommended 27 IHE - Integrating the Healthcare Enterprise (www.ihe.net) - profiles describing the different layers of interoperability at a European level, thus providing software vendors with the appropriate power to develop truly interoperable EHR systems for storing and sharing medical data. Going one-step further, appreciating the greater socioeconomic impact of such endeavor, the EU has acknowledged the necessity of interoperable EHR systems by incorporating them under the umbrella of the Connecting European Facility (CEF) EU instrument towards forming interconnected and trans-European digital infrastructures and networks. Currently ongoing CEF program for cross-border healthcare provision, simultaneously being developed in 17 member states, will facilitate the exchange of the minimum Patient Summary (PS) between countries, while accommodating electronic prescription (ePrescription) and dispensing (eDespensing) functionality, adopting the Directive 2011/24/EU (Joint Action to support the eHealth), by 2020.

Within the context of the ENDORSE project, the objective is to augment our existing EHR system [11] [12], [13] complying with the afore-described interoperability requirements with vital signs data coming from the e-diagnostic support module detailed below. For that purpose, the necessary APIs will be developed relying on the Fast Healthcare Interoperability Resources (FHIR - www.hl7.org/fhir) standard, the most widely used standard for developing smartphone-based mobile health applications today. Lightweight APIs will acquire data coming from different sensors, at preset sampling rates and using the suitable drivers residing in the e-diagnostic modules communication interface, and assemble them into JSON or XML -like format for communicating them to the cloudbased EHR. Naturally, the appropriate security and device authentication procedures will be installed, while the EHR system will facilitate appropriate auditing schemas for registering each communication and data update session. The latter functionality will rely on accumulated experience from FISTAR (future internet for social and technological alignment) and resulting FIWARE-specific enablers that were previously developed. Additional EHR system functionality will allow generating relevant reporting documents, will accommodate statistical analysis, and support export features for further data analytics purposes.

\section{Diagnostic Support Module}

As previously explained, the diagnostic support module will be mounted on the robot chassis allowing the overall robot to become an e-diagnostic mobile station. Equipped with a set of non-invasive sensors/devices (ECG, blood pressure, oximeter, temperature), this mobile station will then be functionally capable of performing medical exams, generating diagnostic reports and securely forwarding them to the patients cloudbased EHR. This module will have to be easily pluggable and benefit from all the existing interfaces available on the robot that are necessary to its own use.

1) Why this module?: The current finding in hospital is that numerous trolleys or carts with medical devices are disseminated in every corridor of the building, often left alone without any supervision. Nurses are losing time searching for them and this can unfortunately happen when they need it urgently. Another risk is that patients having an easy and free access to medical devices on the trolleys can increase the risk of equipments deterioration or degradation. The e-Diagnostic module brings a first answer to this problem gathering in one place and at the right moment all the necessary medical equipment a nurse could need for a medical check-up. Another asset is that all the embedded sensors in the module are already powered on and can be used immediately, which is not the case when all the medical devices are left on a trolley. As all the patients' medical data are instantly sent to a cloud-based server, this also limits human mistakes or loss of papers.

The e-Diagnostic module offers an effective service innovation providing several advantages in terms of:

- Organizational impact: The robot moves towards the medical caller (nurses, doctors) and not the opposite. The e-health module information collection provides diagnostic assistance for healthcare professionals, enabling faster and more efficient management of patients in all circumstances while optimizing the use of specialized human resources.

- Economic impact: All hospitals have budget constraints. Instead of buying multiple medical devices for the same service, the e-diagnostic module gathers all of them in one place allowing thus a gain of time and money. 


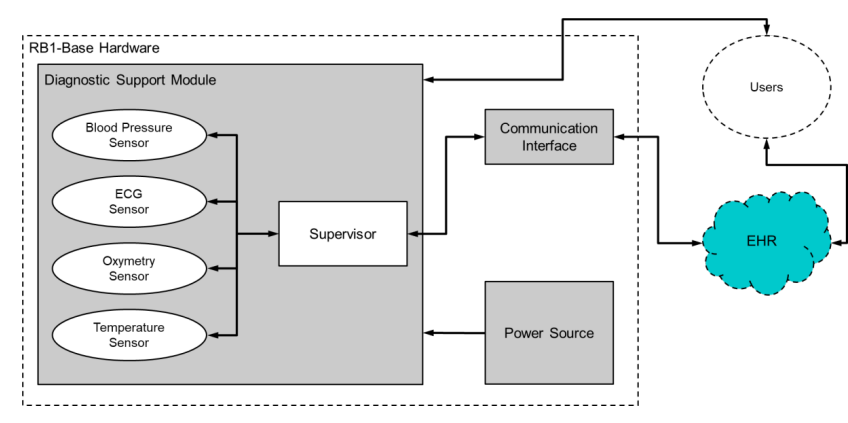

Fig. 3. A schematic view of the e-diagnostic module components and communication interfaces.

- Human impact: Days are already quite long and tedious in a hospital for medical staff having to run everywhere to find their tools. The e-diagnostic module brings simplicity and less physical stress as it moves up to the requester when called.

2) How will it come?: A possible architecture for the diagnostic support module is a pluggable device powered by the robot. This device will consist of a set of non-invasive medical sensors connected to a supervising unit. This unit will be in charge of managing and handling the sensors, but also serve as a preprocessor for the medical data and a gateway to the patients cloud-based EHR. As the robot already features a communication interface, the supervising unit should be able to use this interface to transmit diagnostic reports to the cloudbased EHR.

This whole architecture is illustrated in the block diagram in Fig. 3.

The architecture can be divided into 3 global subsystems:

- Acquisition: A medical data acquisition module represented by its sensors and the supervisor.

- Communication: A communication module represented by heterogeneous networks both intrinsic to the sensors and more generally between the various components of the system.

- Consultation: A consultation module for analysis and diagnosis of results.

\section{CONCLUSION}

ENDORSE is a running three-year project under the umbrella of the Marie Skłodowska-Curie Action (MSCA) Research and Innovation Staff Exchange framework (RISE) Horizon 2020, hence this concept paper described research work-in-progress. ENDORSE implementation will offer a great opportunity to exchange knowledge, know-how and experience between the SMEs and the creative academic groups constituting the ENDORSE consortium. The ultimate goal is the successful research and commercial follow-up emanating from a challenging $R \& D$ topic that intends to address major issues in the industry in general and in healthcare provision in particular.

\section{REFERENCES}

[1] A. G. Ozkil, Z. Fan, S. Dawids, H. Aanes, J. K. Kristensen, and K. H. Christensen, "Service robots for hospitals: A case study of transportation tasks in a hospital," in 2009 IEEE International Conference on Automation and Logistics, Aug 2009, pp. 289-294.

[2] T. Deyle, Why Indoor Robots for Commercial Spaces Are the Next Big Thing in Robotics. IEEE Spectrum, March 2017.

[3] D. Zeinalipour-Yazti and C. Laoudias, "The anatomy of the anyplace indoor navigation service," in ACM SIGSPATIAL Special, ser. SIGSPATIAL'17, vol. 9, no. 2. ACM Press, 2017, book, pp. 3-10.

[4] A. Konstantinidis, P. Irakleous, Z. Georgiou, D. Zeinalipour-Yazti, and P. K. Chrysanthis, "IoT data prefetching in indoor navigation SOAs," ACM Trans. Internet Technol., vol. 19, no. 1, pp. 10:1-10:21, Nov. 2018. [Online]. Available: http://doi.acm.org/10.1145/3177777

[5] C. Combastel, V. Puig, T. Rassi, and T. Alamo, "Special issue: Setmembership methods applied to FDI and FTC," International Journal of Adaptive Control and Signal Processing, no. 30, pp. 147-426, 2016.

[6] N. Ramdani, D. Zeinalipour-Yazti, M. Karamousadakis, and A. Panayides, "Towards robust methods for indoor localization using interval data," in Proceedings of the 20th IEEE International Conference on Mobile Data Management, 2019.

[7] M. H. Amri, Y. Becis, D. Aubry, and N. Ramdani, "Indoor human/robot localization using robust multi-modal data fusion," in 2015 IEEE International Conference on Robotics and Automation (ICRA), May 2015, pp. 3456-3463.

[8] E. Colle and S. Galerne, "A multihypothesis set approach for mobile robot localization using heterogeneous measurements provided by the internet of things," Robotics and Autonomous Systems, vol. 96, pp. 102 $-113,2017$.

[9] "European Commission (2012, Dec.). eHealth Action Plan 2012-2020Innovative Healthcare for the 21st Century: Communication from the commission to the European parliament, the council, the European economic and social committee and the committee of the regions." [Online]. Available: https://ec.europa.eu/digital-single-market/en/news/ ehealth-action-plan-2012-2020-innovative-healthcare-21st-century

[10] "European Commission (2014 Feb.). Green paper on mobile health.” [Online]. Available: http://ec.europa.eu/digital-agenda/en/news/ green-paper-mobile-health-mhealth.

[11] "Deployment of Generic Cross Border eHealth Services in Cyprus, EU research project supported by Grant Agreement number: INEA/CEF/ICT/A2015/11S1451, Action No: 2015-CY-IA-0095."

[12] Z. C. Antoniou, A. S. Panayides, M. S. Neofytou, I. Constantinou, K. Neokleous, E. C. Schiza, C. Loizou, M. Kalakouti-Kassapi, E. Yiapatou, M. Kyriakides, D. Patelis, N. Mpampamis, K. Karkaletsis, A. Berler, C. N. Schizas, and C. S. Pattichis, "Deployment of generic cross border ehealth services in cyprus," IEEE Communication Society eHealth Technical Committee Newsletter, 2017. [Online]. Available: http://ehealth.committees.comsoc.org/files/ 2017/11/2017-SEPT-OCT-.pdf

[13] S. Avgousti, E. G. Christoforou, A. S. Panayides, S. Voskarides, C. Novales, L. Nouaille, C. S. Pattichis, and P. Vieyres, "Medical telerobotic systems: current status and future trends," BioMedical Engineering OnLine, vol. 15, no. 1, p. 96, Aug 2016. [Online]. Available: https://doi.org/10.1186/s12938-016-0217-7 